04

\title{
Применение новых типов многослойных зеркал нормального падения для целей солнечной спектроскопии вакуумного ультрафиолетового диапазона
}

\author{
(C) С.В. Кузин, ${ }^{1}$ А.А. Рева, ${ }^{1}$ С.А. Богачев, ${ }^{1}$ Н.Ф. Ерхова, ${ }^{1}$ Н.Н. Салащенко, ${ }^{2}$ Н.И. Чхало, ${ }^{2}$ В.Н. Полковников ${ }^{2}$ \\ ${ }^{1}$ Физический институт им. П.Н. Лебедева РАН, \\ 119991 Москва, Россия \\ ${ }^{2}$ Институт фризики микроструктур РАН, \\ 603950 Нижний Новгород, Россия \\ e-mail: s.kuzin@lebedev.ru
}

Поступило в Редакцию 3 апреля 2020 г.

В окончательной редакции 3 апреля 2020 г.

Принято к публикации 3 апреля 2020 г.

\begin{abstract}
В настоящее время достигнут значимый прогресс в многослойных зеркалах нормального падения ВУФ диапазона спектра. В первую очередь, это касается повышения коэффициента отражения на рабочей длине волны, уменьшения спектральной ширины кривой отражения, появления высокоэффективных МС покрытий для коротковолнового $(3-9 \mathrm{~nm})$ и длинноволнового (более $50 \mathrm{~nm}$ ) участков спектра. Создание таких зеркал открывает новые возможности для проведения астрофизических исследований Солнца, так как узкие спектральные ширины зеркал в сочетании с высоким коэффициентом отражения позволяют регистрировать изображения солнечной короны в монохроматических линиях. Особенно перспективным является использование телескопов на базе этих зеркал для динамической спектральной диагностики полного диска Солнца методом изображающей спектроскопии. Метод основан на регистрации монохроматических изображений Солнца в линиях ВУФ диапазона спектра с высоким пространственным и временным разрешением. Обсуждается возможный прогресс в солнечных исследованиях с применением этого типа оптики.
\end{abstract}

Ключевые слова: солнечная корона, изображающая спектроскопия.

DOI: 10.21883/JTF.2020.11.49967.113-20

\section{Введение}

Рентгеновская астрономия Солнца занимает одно из ведущих мест в исследованиях физики Солнца. Это связано с тем, что, во-первых, это практически единственный способ прямого изучения протекающих в короне процессов, и, во-вторых, эти исследования имеют важное значение не только для фундаментальных, но и прикладных задач в части космической погоды. За более чем 60 лет космической эры сформировалось несколько выделенных направлений в области рентгеновских исследований короны, одним из которых является изображающая спектроскопия. Это направление сильно связано с развитием экспериментальной базы: детекторов, средств бортовой обработки изображений и многослойных покрытий зеркал нормального падения.

В 90-е года прошлого века был сформулирован и реализован новый метод в космических исследованиях Солнца - метод изображающей спектроскопии. Он подразумевает получение изображений полного диска Солнца одновременно с высоким пространственным и спектральным разрешением. Это связано в первую очередь с необходимостью получить спектроскопическую информацию о взрывных процессах в солнечной короне, необходимую для определения физических характеристик солнечной плазмы: плотности, температуры и меры эмиссии. Так как эти процессы происходят довольно редко, то для повышения вероятности их регистрации необходимо наблюдать полный солнечный диск.

Первый солнечный спектрогелиограф в вакуумной ультрафиолетовой (ВУФ) области спектра функционировал в середине 70-х годов на американской станции SkyLab [1]. В качестве основного одновременно диспергирующего и фокусирующего элемента в нем использовалась сферическая дифракционная решетка квазинормального падения. Вследствие этого прибор имел сравнительно низкую дисперсию, и изображения Солнца в отдельных спектральных линиях, имеющие сравнительно большой угловой размер - 30 arcmin, перенакладывались друг на друга. В середине 90-х годов был достигнут значительный прогресс в развитии высокоэффективных покрытий зеркал нормального падения ВУФ диапазона, что позволило создать спектрогелиограф, в котором функции диспергирующего и фокусирующего элементов были разделены между плоской дифракционной решеткой скользящего падения и широкополосным зеркалом [2]. Несмотря на относительно невысокую дисперсию, прибор реализовал принцип „углового сжатия“ изображения в направлении дисперсии, что позволяет существенно уменышить переналожение спектральных изображений Солнца. 
В середине 90-х годов с появлением новых типов покрытий был реализован другой подход к изображающей спектроскопии полного диска, основанный на одновременной регистрации телескопических изображений солнечной короны в нескольких узких спектральных диапазонах $[3,4]$. В 2009 г. была выведена на орбиту самая мощная на сегодняшний день солнечная лаборатория SDO, включающая многоканальный телескоп AIA [5].

Принципиальное различие между двумя этими подходами заключается в том, что спектрогелиометры строят изображения полного диска Солнца в монохроматических линиях, и, таким образом, предоставляют максимально „чистые“ данные для последующей обработки. В то же время из-за высокой плотности линий в диапазоне $170-350 \AA$ эти изображения или переналожены, или имеют низкое пространственное разрешение в направлении дисперсии, что ограничивает возможности по исследованию компактных вспышечных процессов. Другим ограничением является низкое временное разрешение в следствие невысокой эффективности дисперсионных элементов.

Телескопы лишены этих двух недостатков, однако имеют значительный спектральный диапазон регистрации, и изображение в них формируется большим числом спектральных линий, излучаемых плазмой, находящейся в различных физических условиях. Этот факт сильно усложняет интерпретацию телескопических данных и снижает достоверность получаемых результатов. В последние годы появились новые многослойные покрытия для зеркал нормального падения ВУФ диапазона, обладающие одновременно высокими спектральной селективностью и пиковым коэффициентом отражения. Прогресс в покрытиях такого типа открывает новые возможности для реализации метода рентгеновской спектроскопии. Частично это было реализовано в эксперименте AIA, где спектральные полуширины пропускания каналов составляли (в зависимости от диапазона) от 1 до $15 \mathrm{~nm}$, а высокая пропускающая способность позволила реализовать времена экспозиции несколько секунд.

\section{1. Исследования горячей корональной плазмы}

Регистрация „горячей“ плазмы, с температурами более 5 МК в ВУФ диапазоне обычно проводится в трех диапазонах спектра - 13.2 (линии Fe XXII-XXIII), 19.2 (Fe XXIV) и в последнее время $9.4 \mathrm{~nm}$ (Fe XVI). Однако во всех этих диапазонах горячие линии блендированы, т.е. плохо отделимы более холодными интенсивными линиями, отстоящих от горячих в среднем на $0.02 \mathrm{~nm}$.

По этой причине интересным представляется более коротковолновый диапазон, где плотность холодных линий несколько меньше и может быть достигнута более высокая спектральная селективность покрытий (рис. 1) [6-9].

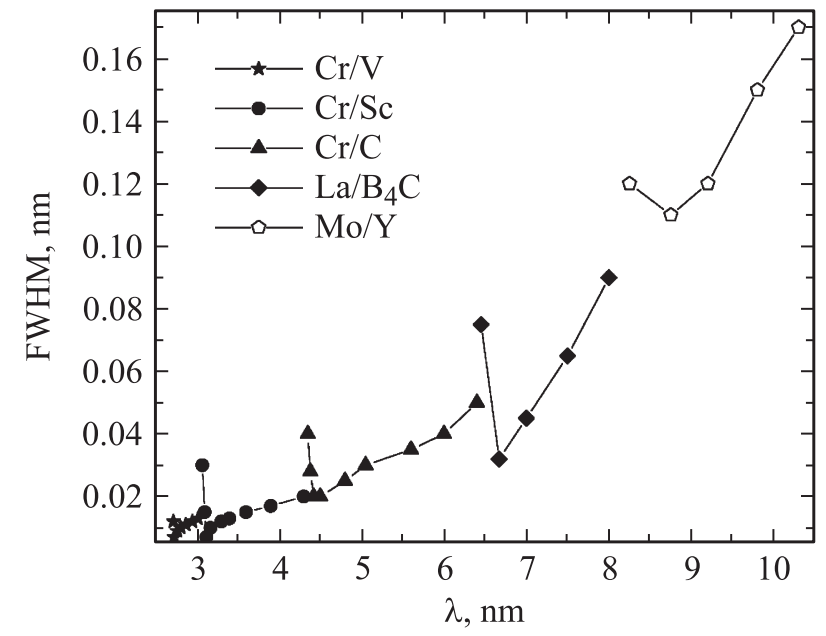

Рис. 1. Спектральная селективность покрытий многослойных зеркал нормального падения в коротковолновой области спектра.

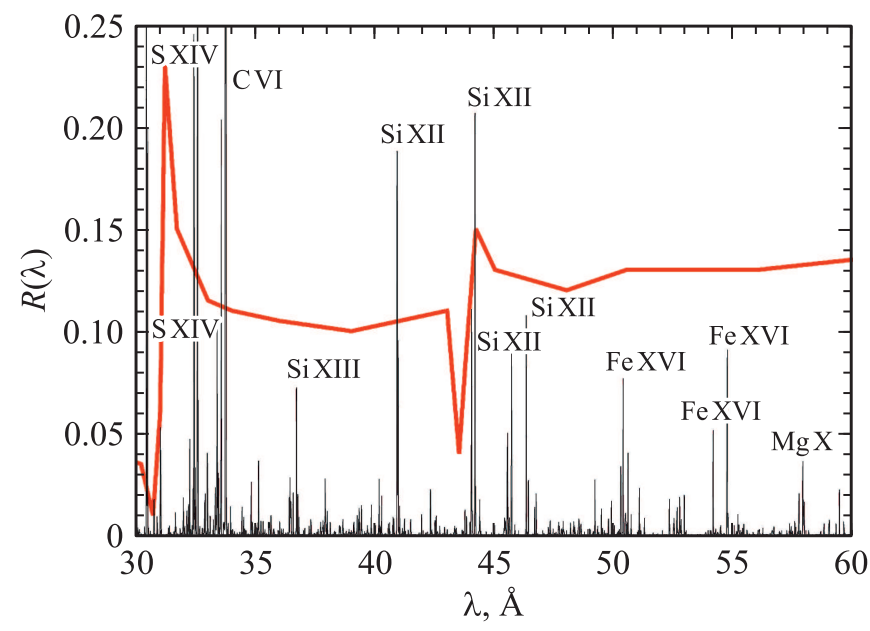

Рис. 2. Спектр Солнца в диапазоне $3-7 \mathrm{~nm}$ и пиковый коэффициент отражения современных покрытий многослойных зеркал нормального падения в коротковолновой области.

В диапазоне 3-6 nm в спектре Солнца есть сильные спектральные линии, часть из которых формируется при высоких температурах. Поэтому есть возможность создания телескопа, который сможет строить монохроматические изображения горячей плазмы на Солнце (рис. 2).

Для этой цели наилучшим образом подходят линии Fe XVI 50.3, 51.0 и $54.7 \AA$ А. В их окрестности нет интенсивных „корональных“ линий с температурами около $1 \mathrm{MK,} \mathrm{и} \mathrm{поэтому} \mathrm{на} \mathrm{изображениях} \mathrm{будет} \mathrm{сигнал} \mathrm{только}$ от горячей плазмы.

\section{2. Регистрация корональной плазмы}

Корональная плазма является наиболее популярным и информативным объектом солнечных исследований. Это 
связано с тем, что в невозмущенной короне преобладает плазма с температурами 0.7-1.2 МК. Она наиболее обильна в короне и наблюдается практически с начала космических исследований Солнца.

Особое значение имеют изображения, полученные в линиях Fe XI-XII. Эти линии формируется при температуре около $0.7 \mathrm{MK,} \mathrm{что} \mathrm{близко} \mathrm{к} \mathrm{средней} \mathrm{температуре}$ короны. Кроме того, железо - наиболее обильный тяжелый элемент короны, поэтому их относительная интенсивность весьма высока. На изображениях короны, полученных в линиях Fe XI-XII, видна структура активных областей, отдельные корональные петли, микроактивность короны. Изображения Fe XI-XII дают косвенную информацию о магнитных структурах на Солнце и их динамике.

Поскольку линии $\mathrm{Fe}$ XI-XII являются одними из сильнейших в спектре Солнца, то их интенсивности излучения достаточно, чтобы наблюдать корону на больших высотах. С небольшими изменениями телескопы, регистрирующие линии Fe XI-XII, можно использовать для наблюдения дальней короны, что важно для исследования корональных выбросов массы.

В то же время сейчас возникли новые задачи, связанные с тонкой диагностикой плазмы и с изучением трансформации энергии в мелкомасштабных процессах в короне. Необходимость получения изображений с высоким пространственным и временным разрешением практически не позволяет использовать спектрогелиометры.

Наиболее перспективной для создания монохроматичного телескопа является линия $\mathrm{Fe}$ XI $17.1 \mathrm{~nm}$. Этот выбор обусловлен двумя причинами: Fe XI $17.1 \mathrm{~nm}$ является одной из самых интенсивных и хорошо изученных корональных линий [10], и этот диапазон очень перспективен с точки зрения монохроматизации. Последнее связано с появлением покрытий многослойных зеркал, имеющих спектральный коэффициент отражения с FWHM $\sim 0.4 \mathrm{~nm}$ в этой области спектра [11]. И одновременно близостью этой линии к скачку поглощения алюминия, являющегося основой фильтров в диапазоне выше $17 \mathrm{~nm}$ [12]. Смещением спектрального положения максимума отражения покрытия зеркала в коротковолновую сторону вблизи $17 \mathrm{~nm}$ можно добиться еще большего увеличения спектральной селективности и таким образом степени монохроматизации, за счет отрезания линий, находящихся за краем поглощения алюминия.

Для этой спектральной области оптимальными покрытиями являются $\mathrm{Be} / \mathrm{Si} / \mathrm{Al}$. Они одновременно обладают и рекордными коэффициентами отражения (до $61 \%$ ) и высокой $(\mathrm{FWHM} \approx 0.4 \mathrm{~nm})$ спектральной селективностью [13]. Этот тип покрытия обладает так же высокой временной стабильностью: наблюдения за рентгенооптическими характеристиками в течение 20 месяцев не обнаружили в пределах экспериментальной ошибки изменения коэффициента отражения и положения брэгговских пиков [14].

\section{3. Регистрация холодной плазмы переходного слоя}

Переходный слой солнечной короны располагается непосредственно над видимой невооруженным глазом фотосферой Солнца. Он крайне важен для изучения, так как именно в нем происходит разогрев плазмы и формирование эруптивных протуберанцев, которые вызывают выбросы корональной массы.

Наблюдения холодной корональной плазмы традиционно проводятся в линии Не II $30.4 \mathrm{~nm}$, имеющей температуру 50-100 kК. С одной стороны, эта линия является наиболее интенсивной в ВУФ диапазоне спектра, с другой она блендирована близкорасположенной корональной линией Si XI (температура - около $1 \mathrm{MK}$ ), отстоящей всего на $0.02 \mathrm{~nm}$, а также рядом еще более горячих и интенсивных линий ионов Fе на длинах волн 28.5 и $33.5 \mathrm{~nm}$. Поэтому в первую очередь встает вопрос о фильтрации именно этих линий. Это может быть осуществлено с применением новых типов покрытий зеркал на основе $\mathrm{Be} / \mathrm{Si} / \mathrm{Al}$ и $\mathrm{Be} / \mathrm{Mg}$, имеющих $\mathrm{FWHM} \sim 1.6 \mathrm{~nm}$ на длине волны $30.4 \mathrm{~nm}$ FWHM, равную 1 и $1.6 \mathrm{~nm}$, и коэффициенты отражения 33 и 56\% соответственно $[11,15]$.

Другой перспективной линией для наблюдения холодной плазмы является линия Не I $58.4 \mathrm{~nm}$. Эта линия возбуждается при температуре $10 \mathrm{kK}$ и соответствует нижней части переходного слоя. Наблюдения солнечной короны в этой линии проводились лишь в одном эксперименте со спектрогелиографом в середине 70-х годов прошлого века [1]. Тем не менее комбинированные наблюдения одновременно в линиях ионов Не I и He II крайне важны, так как позволят детально исследовать процессы, происходящие при разогреве плазмы от фотосферных до корональных температур. Основная проблема, по которой наблюдения в этом диапазоне не проводились практически 40 лет, связана с отсутствием как зеркал нормального падения, так и фильтров на этот диапазон спектра. Однако в ходе подготовки эксперимента „Кортес“ для Международной космической станции [16] в ИФМ РАН были созданы новые типы многослойных покрытий зеркал и фильтры на этот диапазон спектра. Покрытия зеркал на основе структур $\mathrm{Mo} / \mathrm{Mg}$ имеют спектральную ширину $\Delta \lambda \approx 5.5-6 \mathrm{~nm}$ при коэффициенте отражения $R \sim 30 \%$ [17]. Недостатком этой пары является относительно невысокая стабильность коэффициента отражения во времени. У изготовленных покрытий этого типа коэффициент отражения упал примерно на 50\% в течение года наблюдения.

В настоящее время мы приступили к исследованию структуры на основе $\mathrm{Sc} / \mathrm{Si} / \mathrm{Al}$. Первые экспериментальные результаты указывают на перспективность этой структуры. Получен коэффициент отражения более $30 \%$ при лучшей, по сравнению с магнийсодержащими многослойными структурами, временной стабильностью рентгенооптических характеристик. Однако для окончательного вывода о возможности ее использования в 
космических экспериментах требуется провести дополнительный цикл временных исследований.

В качестве фильтров предполагается использование многослойных структур $\mathrm{Al} / \mathrm{Sc}$ [12], эффективно подавляющая второй порядок отражения многослойного зеркала с линией $30.4 \mathrm{~nm}$. Такая комбинация зеркала и фильтра позволяет получить квазимонохроматичное изображение плазмы нижней области переходного слоя.

\section{Заключение}

Прогресс, достигнутый в развитии многослойных структур отражающих покрытий и пропускающих фильтров, позволяет с помощью сравнительно простых инструментов - одно- или двухзеркальных телескопов нормального падения, реализовать метод изображающей спектроскопии полного диска Солнца. Один из первых экспериментов с помощью этого метода будет реализован в ходе эксперимента „Кортес“ на МКС, начало которого запланировано на 2024 г.

\section{Финансирование}

Работа выполнена при поддержке Российского научного фонда (грант 17-12-01567).

\section{Конфликт интересов}

Авторы заявляют, что у них нет конфликта интересов.

\section{Список литературы}

[1] Garrett D.L., Tousey R. // Appl. Optics. 1977. Vol. 16. P. 898-903. DOI: 10.1364/AO.16.000898

[2] Kuzin S.V., Zhitnik I.A., Pertsov A.A., Slemzin V.A., Mitrofanov A.V., Ignatiev A.P., Korneev V.V., Krutov V.V., Sobelman I.I., Ragozin E.N., Thomas R.J. // J. of X-Ray Sci.Technol. 1997. Vol. 7. N 3. P. 233-247.

[3] Delaboudinière J.-P., Artzner G.E., Brunaud J., Gabriel A.H., Hochedez J.F., Millier F., Song X.Y., Au B., Dere K.P., Howard R.A., Kreplin R., Michels D.J., Moses J.D., Defise J.M., Jamar C., Rochus P., Chauvineau J.P., Marioge J.P., Catura R.C., Lemen J.R., Shing L., Stern R.A., Gurman J.B., Neupert W.M., Maucherat A., Clette F., Cugnon P., van Dessel E.L. // Solar Physics. 1995. Vol. 162. N 1-2. P. 291-312. DOI: $10.1007 / \mathrm{BF} 00733432$

[4] Zhitnik I., Kuzin S., Afanas'ev A., Bugaenko O., Ignat'ev A., Krutov V., Mitrofanov A., Oparin S., Pertsov A., Slemzin V., Sukhodrev N., Umov A. // Adv. Space Res. 2003. Vol. 32. N 4. P. 473-477. DOI: 10.1016/S0273-1177(03)00351-X

[5] Title, Alan M. // Bulletin American. Astronom. Society. 2010. Vol. 41. P. 871.

[6] Andreev S.S., Bibishkin M.S., Chkhalo N.I., Kluenkov E.B., Prokhorov K.A., Salashchenko N.N., Zorina M.V., Schafers F., Shmaenok L.A. // J. Synchrotron Radiation. 2003. Vol. 10. P. 358 -360. DOI: $10.1107 / \mathrm{S} 0909049503015255$
[7] Щербаков А.В., Пестов А.Е., Салащенко Н.Н., Торопов М.Н., Чхало Н.И., Полковников В.Н., Клюенков Е.Б., Лопатин А.Я., Лучин В.И., Цыбин Н.Н., Ахсахалян А.Д., Свечников М.В., Нечай А.Н. // Поверхность. Рентгеновские, синхротронные и нейтронные исследования. 2017. № 1. C. 5-24. DOI: 10.7868/S0207352817010048 [Akhsakhalyan A D., Kluenkov E.B., Lopatin A.Ya., Luchin V.I., Nechay A.N., Pestov A.E., Polkovnikov V.N., Salashchenko N.N., Svechnikov M.V., Toropov M.N., Tsybin N.N., Chkhalo N.I., Shcherbakov A.V. // J. Surface Investigation: X-ray, Synchrotron and Neutron Techniques. 2017. Vol. 1. N 1. P. 1-20. DOI: $10.1134 / \mathrm{S} 1027451017010049]$

[8] Барышева М.М., Пестов А.Е., Салащенко Н.Н., Торопов М.Н., Чхало Н.И. // УФН. 2012. Т. 182. № 7. C. 727-747. DOI: $10.3367 /$ UFNr.0182.201207c.0727

[Barysheva M.M., Pestov A.E., Salashchenko N.N., Toropov M.N., Chkhalo N.I. // Phys.-Uspekhi. 2012. Vol. 55. N 7. P. 681-699. DOI: $10.3367 /$ UFNe.0182.201207c.0727]

[9] Chkhalo N.I., Künstner S., Polkovnikov V.N., Salashchenko N.N., Schäfers F., Starikov S.D. // Appl. Phys. Lett. 2003. Vol. 102. N 1. P. 11-14. DOI: 10.1063/1.4774298

[10] Кузин С.В., Богачев С.А., Житник И.А., Шестов С. В., Слемзин В.А., Митродбанов А.В., Суходрев Н.К., Перцов А.А., Игнатьев А.П., Бугаенко О.И., Иванов Ю.С., Рева А.А., Зыков М.С., Ульянов А.С., Опарин С.Н., Гончаров А.Л., Шергина Т.А., Урнов А.М., Соловьев В.А., Попова С.Г. // Известия РАН. Сер. физическая. 2010. T. 74. C. 39-41. [Kuzin S.V., Bogachev S.A., Zhitnik I.A., Shestov S.V., Slemzin V.A., Mitrofanov A.V., Sukhodrev N.K., Pertsov A.A., Ignat'ev A.V., Ivanov Yu.S., Reva A.A., Zykov M.S., Ul'yanov A.S., Oparin S.N., Goncharov A.L., Shergina T.A., Urnov A.M., Solov'ev V.A., Popova S.G., Bugaenko O.I. // Bulletin of the Russian Academy of Sciences: Physics. 2010. Vol. 74. N 1. P. 33-37. DOI: $10.3103 / \mathrm{S} 1062873810010090]$

[11] Chkhalo N., Lopatin A., Nechay A. Pariev D., Pestov A., Polkovnikov V., Salashchenko N., Schäfers F., Sertsu M., Sokolov A., Svechnikov M., Tsybin N., Zuev S. // J. Nanosci. Nanotechnol. 2019. Vol. 19. P. 546-553. DOI: $10.1166 /$ jnn.2019.16474

[12] Chkalo N.I., Drozdov M.N., Kluenkov E.B., Kuzin S.V., Lopatin A.Ya., Luchin V.I., Salashchenko N.N., Tsybin N.N., Zuev S.Yu. // Appl. Optics. 2016. Vol. 55. P. 4683-4690. DOI: $10.1364 / A O .55 .004683$

[13] Chkhalo N.I., Pariev D.E., Polkovnikov V.N., Salashchenko N.N., Shaposhnikov R.A., Stroulea I.L., Svechnikov M.V., Vainer Yu.A., Zuev S.Yu. // Thin Solid Films. 2017. Vol. 631. P. 106-111. DOI: 10.1016/j.tsf.2017.04.020

[14] Полковников В.Н., Салащенко Н.Н., Свечников М.В., Чхало Н.И. // УФН. 2020. Т. 190. С. 92-106. DOI: $10.3367 /$ UFNr.2019.05.038623

[15] Polkovnikov V.N., Chkalo N.N., Pleshkov R., Salashchenko N.N., Schäfers F., Sertsu M.G., Sokolov A., Svechnikov M.V., Zuev S.Yu. // Opt. Lett. 2019. Vol. 44. N 2. P. 263-266. DOI: 10.1364/OL.44.000263

[16] Shestov S.V., Ulyanov A.S., Vishnyakov E.A., Pertsov A.A., Kuzin S.V. // Proceedings of the SPIE. Space telescopes and instrumentation 2014: Ultraviolet to Gamma ray. 2014. Vol. 9144. id. 91443G. DOI: 10.1117/12.2055946

[17] Bogachev S.A., Chkhalo N.I., Kuzin S.V., Pariev D.E., Polkovnikov V.N., Salashchenko N.N., Shestov S.V., Zuev S.Y. // Appl. Optics. 2016. Vol. 55. N 9. P. 2126-2135. DOI: $10.1364 / A O .55 .002126$ 